\title{
Load-sharing strategy taking account of compressor degradation*
}

\author{
Marta Zagorowska ${ }^{1}$, Nina Thornhill ${ }^{2}$, Trond Haugen ${ }^{3}$, and Charlotte Skourup ${ }^{4}$
}

\begin{abstract}
The objective of designing a control structure that takes the degradation of the system into account is to preserve its performance and mitigate further damage. This problem is often encountered in process industries, e.g. in gas processing plants, where the question arises how to distribute the control effort among multiple actuators based on their degradation. The main focus of this work is to investigate how to assign the loads in a two-compressor system taking the degradation, i.e. the loss of available performance, into consideration. Contrarily to other approaches, such as methods based on distance to surge or predictive control, the algorithm proposed in this work does not require a reconfiguration of the control structure, at the same time taking explicitly the degradation into account. The simulation results confirm that this approach mitigates further loss of performance, in particular for compressors, which have significantly different degradation rates.
\end{abstract}

\section{INTRODUCTION}

The concept of designing a control structure taking into account the degradation of the system has been described by Ray et al. in [1]. The attenuation of the detrimental changes, which are induced both by ageing and varying operating conditions [2], is sought in order to keep the available performance of the system. In particular, Härkegård and Glad, [3], analysed the question of distributing the control effort in a multi-actuator system. Such systems are encountered in process industries, e.g., in the oil and gas processing plants where the distribution of the control effort between the machines while preserving the safety constraints is of importance. In case of turbomachinery, such as pumps and compressors, the control effort is called the load and denotes the amount of fluid processed by a single piece of equipment. This work proposes a load-sharing algorithm for a two-compressor system based on a degradation indicator.

A load-sharing algorithm for centrifugal compressors was described in [4] and is based on the assumption that the compressors, which are not identical, should work at the same distance to surge. The load is assigned proportionally. The approach based on proportional load assignment was

*Financial support is gratefully acknowledged from the Marie Curie Horizon 2020 EID-ITN project "PROcess NeTwork Optimization for efficient and sustainable operation of Europe's process industries taking machinery condition and process performance into account PRONTO", Grant agreement No 675215.

${ }^{1}$ Marta Zagorowska is with Department of Chemical Engineering, Imperial College London, South Kensington, SW7 2AZ London, UK, m. zagorowska@imperial.ac.uk

${ }^{2}$ Nina Thornhill is with Department of Chemical Engineering, Imperial College London, South Kensington, SW7 2AZ London, UK, n.thornhilleimperial.ac.uk

${ }^{3}$ Trond Haugen is with ABB AS, Ole Deviks vei 10, 0666, Oslo, Norway trond. haugen@ no. abb. com

${ }^{4}$ Charlotte Skourup is with ABB AS, Ole Deviks vei 10, 0666, Oslo, Norway charlotte.skourupdno.abb.com a starting point for this paper. The framework based on distance to surge was used also by companies [5], [6] and more recently by [7], [8], and is available in industrial controllers, e.g., [9], [10]. In [11], [12] a more advanced framework was proposed based on predictive control. It combines a load-sharing algorithm with a surge protection scheme. As an optimization-based framework, it is also able to take additional constraints into account. Another optimization-based framework was proposed in [13], where steady-state calculations were employed to schedule the loads for maintenance purposes, including also the timerelated degradation, but only in a steady-state framework. This work combines the degradation attenuation with the load-sharing problem.

The paper is structured as follows. In the next section, a multi-compressor system is described, including a model of a single compressor train. Section III introduces the degradation model, analyses its influence on the parameters of the system, and proposes a modification based on the operating conditions. The load-sharing algorithm taking the degradation into account is developed in Section IV. The case study used for this work as well as the numerical values for the degradation model are presented in Section V. Section V-B describes the results of the simulation in three scenarios: no degradation, one compressor degrading, and both compressors degrading. Finally, the conclusions and a proposition of further research are included.

\section{COMPRESSOR MODELLING}

The control system analysed in this work consists of two compressors with Variable Speed Drives (VSD), a discharge tank, and a suction tank (Fig. 1) [12]. The equations for the pressures are derived from mass balances in each tank:

$$
\begin{aligned}
& p_{d}=\frac{a_{01}^{2}}{V_{d}}\left(m-m_{\text {out }}\right) \\
& p_{s}=\frac{a_{01}^{2}}{V_{s}}\left(m_{\text {in }}-m\right)
\end{aligned}
$$

where $m$ is the sum of the mass flows through the compressors, $m=m_{1}+m_{2}, m_{\text {in }}$ is the inlet mass flow, $m_{\text {out }}$ is the outlet mass flow. The constants are: $a_{01}$ - speed of sound, $V_{s}, V_{d}$ - volume of the suction and discharge tanks.

The control structure in the system is as follows. The flow is controlled downstream of the compressors, so there is no independent flow controller in Fig. 1. The suction pressure is controlled by adjusting the opening of the inlet valve (PI control). The discharge pressure is controlled by adjusting the speed of the compressors. To keep the schematics simple, 


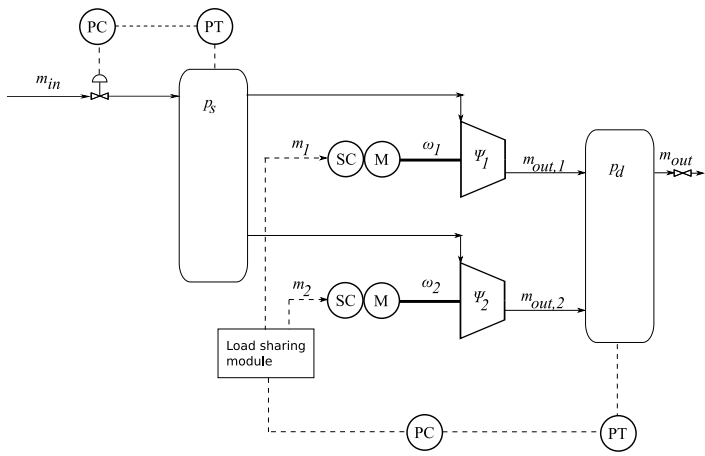

Fig. 1: A control system consisting of two compressors with Variable Speed Drives (VSD), a suction and a discharge tank

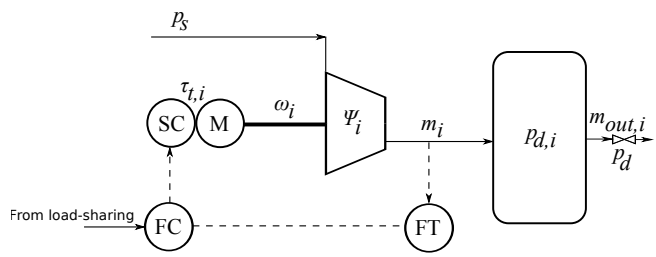

Fig. 2: Single compressor train consisting of a compressor with a VSD and a discharge tank representing the passages inside the system

Fig. 2 depicts separately a single compressor train with a slave flow controller.

Gravdahl in [14] analyzed a single compressor as shown in Fig. 2 consisting of a compressor described by a static compressor map $\Psi_{i}$, a discharge tank with volume $V_{d, i}$, and an electric motor providing the torque $\tau_{t, i}$ to the compressor to achieve the speed $\omega_{i}$. The dynamics of the system are described with a set of nonlinear ordinary differential equations describing the mass balance in the tank (Equation (2a)), the mass flow through the compressor (Equation (2b)), the torque balance of the compressor shaft (Equation (2c)):

$$
\begin{aligned}
\dot{p}_{d, i} & =\frac{a_{01}^{2}}{V_{d, i}}\left(m_{i}-m_{\text {out }, i}\right) \\
\dot{m}_{i} & =\frac{A_{1}}{L_{c}}\left(\Psi_{i}\left(m_{i}, \omega_{i}\right) \cdot p_{s}-p_{d, i}\right) \\
\dot{\omega}_{i} & =\frac{1}{J_{i}}\left(\tau_{t, i}-\tau_{c, i}\left(m_{i}, \omega_{i}\right)\right)
\end{aligned}
$$

where $p_{d, i}$ - pressure in the $i$-th discharge tank, $m_{i}$ - mass flow rate through the compressor, $\omega_{i}$ - compressor speed. Moreover, $\Psi_{i}$ denotes the compressor map describing the pressure ratio as a function of mass flow rate and compressor speed [15], $\tau_{c, i}$ - reaction torque of the compressor. The mass flow through the outlet $m_{\text {out }, i}$ valve is a function of the valve opening $u_{\text {out }, i}$. The variable $\tau_{t, i}$ denotes the external torque delivered to the compressor. The constants are: $V_{d, i}$ - volume of the discharge tank, $A_{1}$ and $L_{c}$ - the geometric parameters of the internal piping system, $J_{i}$ - the inertia of the shaft.

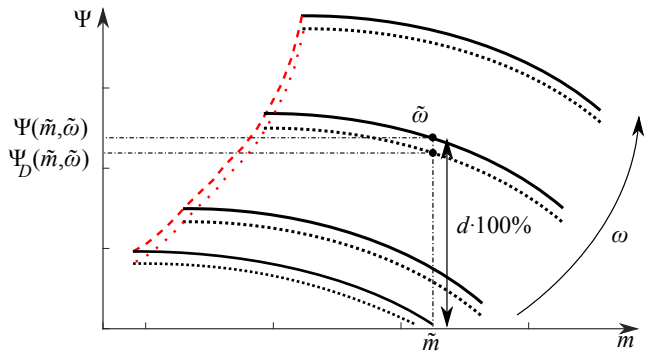

Fig. 3: A compressor map $\Psi$ (solid black lines) with the surge line (dashed red line) changes with degradation $d$ and becomes $\Psi_{D}$ (dotted black lines) with the shifted surge line (dotted red line) [18]. The point $\tilde{m}, \tilde{\omega}$ denotes the steadystate operating point for undegraded compressor

\section{DEGRADATION MODELLING}

A common cause of degradation is a result of fouling of compressor blades and roughening of blade surfaces, both of which lead to deterioration of compressor performance as shown experimentally in [16]. According to [17], the loss of the pressure ratio caused by degradation can be described using the compressor characteristics $\Psi(m, \omega)$ :

$$
\Psi_{D}(t, m(t), \omega(t))=d(t) \cdot \Psi(m(t), \omega(t))
$$

where $\Psi_{D}$ denotes the characteristics of the degraded equipment and $d(t)$ denotes the degrading health, $d(0)=1$ denotes perfect health (see, e.g., [17] or [18]). Moreover, as $\Psi_{D}$ represents a compressor map, $\Psi_{D} \geq 1$ for all $m$ and $\omega$. [17] suggests also that the health loss $1-d(t)$ is not larger than $3-4 \%$ then it is saturated and does not increase further. Figure 3 presents in a graphical way how degradation influences the compressor map by shifting the speed lines [19].

The degradation from [17] and [18] depends solely on time and can be linked with the fouling phenomenon. For $i$-th compressor it is modelled as an exponential function of form:

$$
d_{T, i}(t)=\alpha+\beta \exp \left(-\gamma_{i} t^{2}\right)
$$

where $\alpha>0, \beta>0$, and $\gamma_{i}>0$ are constant parameters defining the maximal degradation level and the slope. The subscript $T$ emphasises the time-dependence of the degradation function.

\section{A. Influence of the degradation in a closed-loop system}

Introducing the degradation of form (3) changes the behaviour of the system, as the resulting pressure ratio will be smaller for the same mass flow and compressor speed. Let $\tilde{m}, \tilde{\omega}$ denote the steady-state mass flow and compressor speed in undegraded state, and let $\tilde{m}_{D}, \tilde{\omega}_{D}$ denote the steady-state mass flow and compressor speed in a system with degradation. To keep the desired discharge pressure and distance to surge, the pressure controller tries to compensate for the loss of the pressure ratio in a degraded compressor. According to Equation (3):

$$
\Psi_{D}(\tilde{m}, \tilde{\omega})=d \cdot \Psi(\tilde{m}, \tilde{\omega})
$$


and

$$
\Psi_{D}\left(\tilde{m}_{D}, \tilde{\omega}_{D}\right)=d \cdot \Psi\left(\tilde{m}_{D}, \tilde{\omega}_{D}\right)
$$

Then to keep $\Psi_{D}\left(\tilde{m}_{D}, \tilde{\omega}_{D}\right)=\Psi(\tilde{m}, \tilde{\omega})$ the following equality must be fulfilled:

$$
\Psi_{D}\left(\tilde{m}_{D}, \tilde{\omega}_{D}\right)=d \cdot \Psi\left(\tilde{m}_{D}, \tilde{\omega}_{D}\right)=\Psi(\tilde{m}, \tilde{\omega})
$$

Taking into account the assumption $0<d \leq 1$ gives:

$$
\Psi\left(\tilde{m}_{D}, \tilde{\omega}_{D}\right)=\frac{\Psi(\tilde{m}, \tilde{\omega})}{d} \geq \Psi(\tilde{m}, \tilde{\omega})
$$

There are then two ways to increase the pressure ratio: by decreasing the mass flow for constant speed, $\tilde{m}_{D}>\tilde{m}$ (moving along the speed lines in Fig. 3), or by increasing the speed for constant mass flow, $\tilde{\omega}_{D}>\tilde{\omega}$ (moving upwards in Fig. 3) which was chosen for this work.

\section{B. Modifying factor}

The authors of [20] suggest that the deviations of the speed of the compressor are one of the main factors contributing to the degradation. Therefore a modifying factor is introduced in Eq. (3):

$$
d_{i}(t)=d_{T, i}(t) \cdot d_{\tau, i}\left(\tau_{t, i}(t)\right)
$$

where $\tau_{t, i}$ denotes the overall torque applied to the $i$-th compressor. Focusing on the torque as the main factor influencing the degradation allows a more direct analysis of the influence of control on degradation as it bypasses the dynamic effect introduced by the inertia of the compressor (Eq. (2c)).

To preserve the properties of $d_{i}(t)$, i.e., $d_{i}(t)<1$ and $d_{i}(t)$ bounded, an exponential modifier was introduced:

$$
d_{\tau, i}\left(\tau_{t, i}(t)\right)=a+b \exp \left(-c_{i}\left(\tau_{t, i} / r_{i}\right)^{2}\right)
$$

The formula (10) is similar to (4), i.e., the baseline degradation model is an exponential, lower-bounded function. Both $d_{T, i}$ and $d_{\tau, i}$ are strictly decreasing functions with respect to their arguments, i.e., time $t$ and torque $\tau_{t, i}$. However, as the applied torque $\tau_{t, i}$ is a combination of the load-sharing algorithm and the discharge pressure PID control, it varies with time, decreasing or increasing depending on the error in the discharge pressure. Therefore, $d_{\tau, i}$ is non-monotonic with respect to time if $\tau_{t, i}(t)$ is a non-monotonic function of time. Hence it becomes an accelerating factor: if $\tau_{t, i}$ increases, the overall degradation indicator $d_{i}$ will decrease faster, because $d_{\tau, i}$ will be smaller; if $\tau_{t, i}$ decreases, then $d_{\tau, i}$ will increase, because it is a decreasing function of $\tau_{t, i}$, and in consequence, $d_{i}$ will decrease more slowly.

\section{LOAD-SHARING TAKING DEGRADATION INTO ACCOUNT}

The objective of this work is to propose a load-sharing method which takes the current degradation level into account and attempts to mitigate further degradation. Assuming that the compressors are similar, i.e., of the same type and configuration, and have the same characteristics, the main approach to load-sharing is based on keeping a selected parameter equal for both compressors, usually the distance to surge [4]. Based on the ratio $\frac{K_{2}}{K_{1}}$ where $K_{i}$ denotes the surge set-point parameters for $i$-th compressor, the load is proportionally scaled according to formula: (11)

$$
m_{2}=m_{1} \frac{K_{2}}{K_{1}}
$$

where $m_{i}$ is the load assigned to $i$-th compressor, e.g. $m_{1}=\frac{m}{2}$ where $m$ is the inlet flow from the upstream side. The formula (11) allows a certain flexibility in the load distribution, however, since it is based on set-points rather than on measurements, it does not take into account the possible changes in the system due to degradation.

This work proposes to assign the loads according to (12):

$$
\begin{aligned}
& m_{1}=\frac{m}{2} \exp \left(q\left(1-f\left(d_{1}, d_{2}\right)\right)\right) \\
& m_{2}=m-m_{1}
\end{aligned}
$$

where $d_{i}$ denotes the current degradation level of $i$-th compressor and $q>0$ is a scaling factor. The function $f(\cdot)$ was chosen as $f\left(d_{1}, d_{2}\right)=\frac{d_{2}}{d_{1}}=d$, because it was assumed that the second compressor has a slower degradation rate. Hence $f\left(d_{1}, d_{2}\right)>1$ and the exponent in Eq. (12) becomes negative. The equation (12) does not depend on the form of $f$, nor $d_{i}$. The form of the function $f\left(d_{1}, d_{2}\right)$ proposed in this work means that always when $d_{1}=d_{2}$, the compressors will get equal load, i.e. the Eq. (12) becomes equivalent to equal load distribution with

$$
m_{1}=m_{2}=\frac{m}{2}
$$

The scaling factor $q$ indicates how much less load the more degraded compressor will receive depending on the current degradation ratio $d=\frac{d_{1}}{d_{2}}$. The choice of $q$ and its influence on the system is now analysed as the load-sharing algorithm provides the set-points for internal flow controllers, and therefore, contributes to the torque calculations. There are two things to consider while choosing the parameters of the algorithm: minimal admissible load and influence of the load-sharing on degradation. The rest of this section presents the analysis of those issues.

\section{A. Minimal admissible load}

The minimal admissible load describes the minimum values of mass flow that guarantee that the compressor stays on the right side of the surge line in Fig. 3. The minimum value for $i$ th compressor is assumed $m_{i}^{m i n}$. This means that (from Eq. (12)):

$$
\begin{aligned}
& m_{1}=\frac{m}{2} \exp (q(1-d))>m_{1}^{\text {min }} \\
& m_{2}=m-m_{1}>m_{2}^{\text {min }}
\end{aligned}
$$

for all values of $d$ with the inlet mass flow $m$. Hence:

$$
\exp (q(1-d))>\frac{2 m_{1}^{\min }}{m}
$$

The right side of the inequality (15) shows that the loadsharing algorithm cannot assign less than a certain fraction of equally distributed load to the degraded compressor. It means that the degraded compressor cannot get less than:

$$
m_{1}^{\min , \%}=\frac{2 m_{1}^{\min }}{m} \cdot 50 \%
$$


of the overall mass flow $m$ taking equal load assumption as default.

On the other hand, the function $\exp (q(1-d))$ is a strictly decreasing function of $d$, so it takes minimal value for maximal $d$. Since $d=\frac{d_{2}}{d_{1}}$, it will be maximal if $d_{2}$ takes maximal value, i.e., $d_{2}=d_{2}^{\max }$ and $d_{1}$ takes minimal value, $d_{1}=d_{1}^{\text {min }}$. Then $d^{\text {max }}=\frac{d_{2}^{\text {max }}}{d_{1}^{\text {min }}}$ and solving the inequality (15) for $q$ with $d=d^{\max }$ gives the upper boundary $q<q^{\max }$.

\section{B. Influence of load-sharing on degradation}

As described in Section III-A, the degradation influences the closed-loop system by forcing it to increase the speed and, in consequence, the torque applied to the compressor, to compensate for loss of performance. This results in increased modifying factor $d_{\tau, i}\left(\tau_{t, i}(t)\right)$, which accelerates the degradation $d_{i}\left(t, \tau_{t, i}(t)\right)$. It would require introducing some additional limits on the slave flow controller to mitigate this influence, however, this implies a reconfiguration of the single compressor control structure. Nonetheless, the loadsharing formula also contributes to the torque calculation and its output depends on the scaling factor $q$. Using again the fact that the function $\exp (q(1-d))$ is a strictly decreasing function of $d$ and calculating its derivative shows that $q$ indicates the rate with which the load will be assigned to the degraded compressor, i.e., larger $q$ means faster achievement of a lower load on the degraded compressor, or equivalently, a higher load on the healthier one. In consequence, the healthier compressor will degrade faster for a larger $q$. On the other hand, $q$ should be sufficiently large to amplify the influence of degradation ratio.

\section{Simulation}

This section describes the compressors used for the simulation and presents the results of the load-sharing algorithm. The proposed algorithm is validated on a Simulink model based on the experimental data from [21], [22] and [23]. The system from Fig. 1 was implemented using Eq. (1)(2c). The data come from the experimental system reported in Cortinovis et al. [22], who analysed a control system for a single compressor. It is assumed that the geometrical parameters of the compressor, as well as the volumes of the intermediate discharge tanks and the inertia of the shaft are the same in both compressors. The set-points for pressures were chosen as: $p_{d}=1.1 \mathrm{bar}, p_{s}=0.9865$ bar. The external pressure is assumed to be $p_{\text {out }}=1.01$ bar.

The compressor map used for this study was calculated using third order polynomial approximation [24] and can be found in Fig. 4a (based on [22], [21], and [23]). It is assumed that, when undegraded, both compressors have identical characteristics, i.e. $\Psi_{1}=\Psi_{2}$.

The other static models include the characteristics of the valves, which relate the valve opening and the pressure drop across the valve to the mass flow, and the torque characteristics for the compressor, which describe the reaction torque $\tau_{c, i}$ as a function of the mass flow and the compressor speed. They were approximated using third and second order

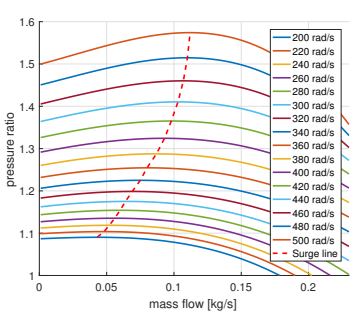

(a) Compressor map $\Psi_{i}$ with surge line

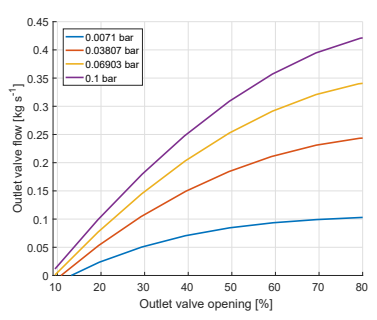

(b) The outlet valve map

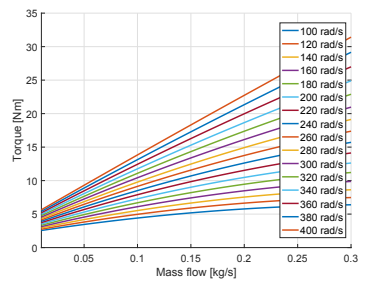

(c) Torque map

Fig. 4: Characteristics of the system based on [21], [22], [23]

polynomials, respectively, with parameters based on [23], [22], and [21] and are depicted in Fig. 4.

\section{A. Degradation case study}

The degradation was introduced to the case study in form of Eq. (3) with $d_{i}$ given by Eq. (9) with parameters $\alpha=0.97$, $\beta=0.03, \gamma_{1}=0.0005, \gamma_{2}=0.00005, a=0.97, b=0.03$, $c_{1}=0.009, c_{2}=0.00005, r_{1}=3, r_{2}=2$. The values were chosen arbitrarily to ensure that $d_{i}(t) \rightarrow 97 \%$ as $t \rightarrow \infty$ and that the degradation rate is slower for the second compressor. It is assumed that there is no torque related degradation at the beginning of the simulation, i.e. the modifying factor $d_{\tau, i}\left(\tau_{t, i}(0)\right)=1$. As the system (2) is stationary, i.e. all the parameters, bar the compressor map $\Psi(m, \omega)$, do not depend on time, and there are no external disturbances, the torque is influenced only by the set-point changes from the load-sharing algorithm. While a real compressor would typically degrade over 2000 hours of operation [18], the simulations in this paper used a much shorter timescale in order facilitate the exploration of the concepts. Therefore, the degradation function was accelerated to stabilise at 200 s. The parameters of (9) can be found using the approach proposed in [17] which is based on fitting the exponential curve to measurements.

Solving the inequality (15) for $q$ with $m=0.38, m_{i}^{\text {min }}=$ $0.12, d_{1}^{\min }=0.97, d_{2}^{\max }=1$ gives $q<q^{\max }=13.9$. To preserve the safety margin, in this work $q=10$. Equation (16) gives $m_{1}^{\min , \%}=0.65 \cdot 50 \%=32 \%$ of $m$ as minimal admissible load.

On the other hand, Fig. 5 shows the modifying factors for both compressors with respect to $q$ - the values close to 13 have a significant influence on the degradation, whereas the values close to one keep the degradation at a similar level. The value $q=10$ provides a trade-off between accelerating 


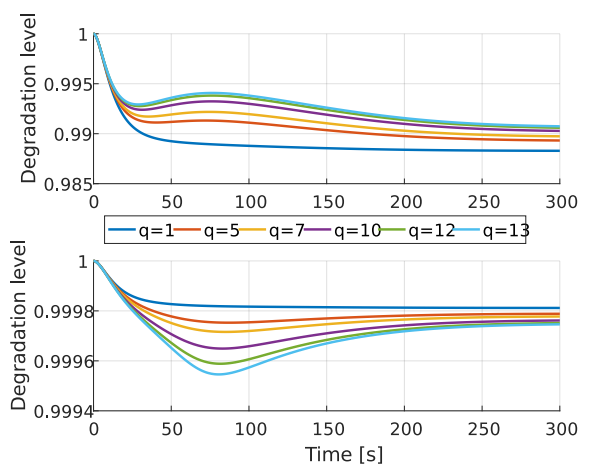

Fig. 5: Analysis of the function $\exp (q(1-d))$

the degradation and amplifying the degradation ratio for load-sharing.

\section{B. Results}

Three scenarios are evaluated in this section. The first one shows the behaviour of the whole system if there is no degradation, i.e. $d_{1}=d_{2}=1$. Therefore, the formulas (12) and (13) are the same. The first one assumes that one compressor is degrading, whereas the other one remains in perfect health, i.e., $d_{1}=d_{1}\left(t, \tau_{t, 1}\right), d_{2}=1$. This situation appears in practice if an already operating compressor with degradation indicator $d_{1}$ is combined with a new (or a refurbished) one with the indicator $d_{2}$. The last case focuses on two degrading machines with different degradation rates, which reflects a typical situation in a multi-compressor system. The case where they both have the same degradation rates yields $\frac{d_{2}}{d_{1}}=1$ regardless of the degradation and the formula (12) becomes equal load sharing (13), and was therefore omitted.

1) Scenario with no degradation: The first simulation was conducted to confirm that the system worked as expected, i.e. the pressure controllers for suction and discharge tanks as well as the flow controllers for each compressor were properly tuned and ensured steady-state operation. After a short transient, the pressure goes into steady-state. The mass flows through the compressors were equal to their set-points.

2) Scenario with one degraded compressor: The second simulation was based on the assumption that only one compressor degrades, whereas the other one is in perfect condition $d_{2}(t)=1$. Assuming that the loads are equal, both compressors are following the same set-points for their mass flows. According to Eq. (12), the healthy compressor should get proportionally more load than the degraded one. To keep the discharge pressure set-point and the mass flow set-point derived from equal load calculation, the actual load for the degraded compressor increases (blue line in Fig. 8a). Therefore, according to the degradation formula (9), the health of the compressor decreases (blue line in Fig. 8b).

However, the load-sharing based on degradation is able to slow down the health decrease induced by the load (Eq. (10)). The set-point for degraded compressor decreases (thick yellow line in the middle of Fig. 6), whereas the healthy one gets more load (thick yellow line in the bottom of Fig. 6). Therefore, the actual load for the degraded compressor decreases as well (yellow line in Fig. 8a, purple line denotes the load for the healthy compressor). This approach makes the degradation indicator slow down (yellow line in Fig. 8b).

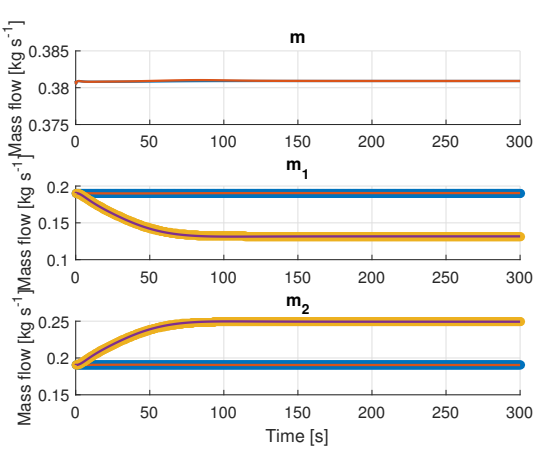

Fig. 6: Mass flow if the first compressor degrades. The top figure presents the inlet flow, whereas the middle and bottom ones show the flows through the first and second compressor
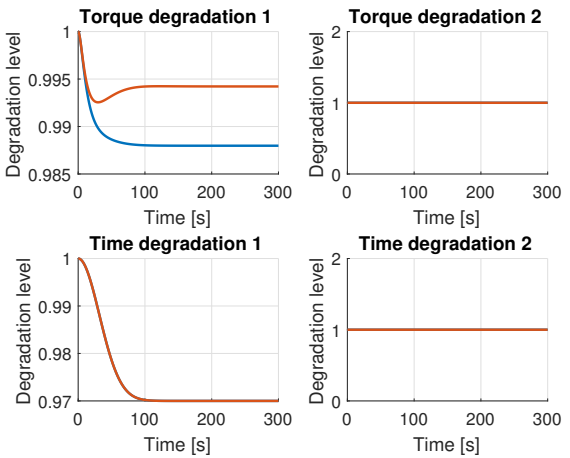

Fig. 7: Degradation types if the first compressor degrades

Further analysis of the degradation indicator is depicted in Fig. 7, where the top plots show the degradation induced by load and the bottom plots present time dependent degradation. The bottom plots are the same in case of equal load-sharing and load-sharing based on degradation. The first compressor degrades until the saturation at 0.97 is reached, whereas the second compressor remains in perfect health. It is also assumed that there is no load-induced degradation in the second compressor (top right plot in Fig. 7). However, the left plot in Fig. 7 shows that the load-induced degradation in the first compressor was smaller if the degradationbased load-sharing (red) was used. This confirms that the proposed algorithm is able to mitigate the degradation, while preserving the values of the discharge pressure.

3) Scenario with two degrading compressors: The loadsharing proposed by Eq. (12) was able to slow down the degradation by decreasing the requirements for the degraded compressor while increasing the set-points for the healthy one. However, the assumption that only one compressor 


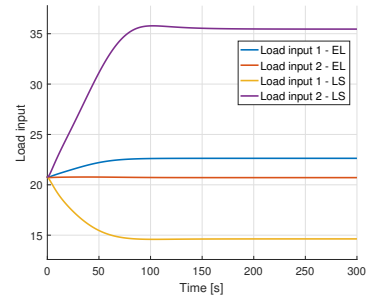

(a) Torque

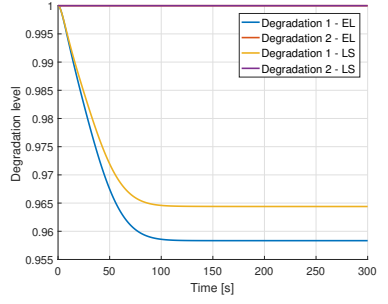

(b) Degradation
Fig. 8: Applied torque and degradation if the first compressor degrades

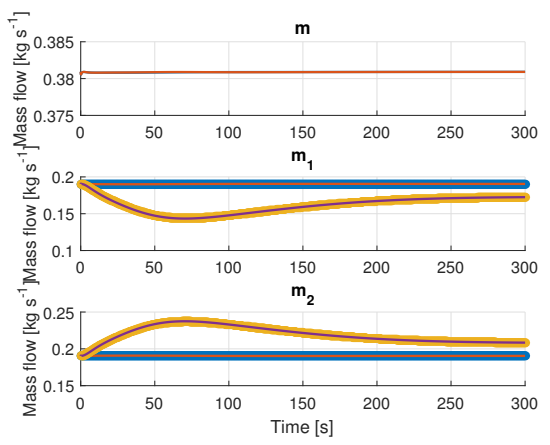

Fig. 9: Mass flow if both compressors degrade. The top figure presents the inlet flow, whereas the middle and bottom ones show the flows through the first and second compressor

decreases was a simplification. This section analyzes the behaviour of the load-sharing algorithm when both compressors are degrading, however at different rates: the first compressor degrades in the same way as described in Section V-B.2, whereas the degradation rate $d_{2}$ of the second one is slower.

Since the first compressor is more degraded, $d_{1}<d_{2}$, it gets initially less load, while the other one gets more (yellow lines in Fig. 9, in the middle and bottom plot, respectively). However, increased load accelerates the degradation of the second compressor, which means that $\frac{d_{2}}{d_{1}} \rightarrow 1$ and back to equal load. Therefore the load for the first compressor increases, whereas the load for the second one decreases (yellow and purple lines in Fig. 11a). This results in further degradation of the first compressor and a slowdown of the degradation of the other one (Fig. 11b). However, as the indicators are bounded functions of the input, the degradation in both cases saturates at the given level. It can be observed, nonetheless, that the algorithm was able to slow down the degradation of the first compressor compared to equal load approach (yellow line against the blue line in Fig. 11b).

Analysis of Fig. 10 suggests that the proposed algorithm is also valid if both compressors are degrading at different rates. It was assumed that the second compressor degrades slower than the first one, to preserve the element $\frac{d_{2}}{d_{1}}$ in Eq. (12) above unity. The time-related degradation, as independent of process variables, remains the same as in section V-B.2 (bottom plots in Fig. 10). The top plots in Fig. 10 show that the algorithm based on Eq. (12) is able to mitigate
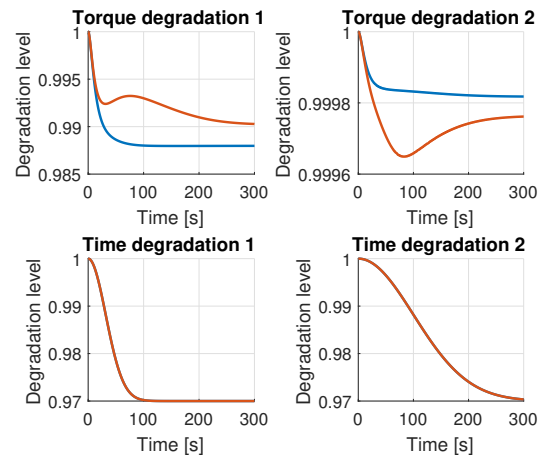

Fig. 10: Degradation types if both compressors degrade

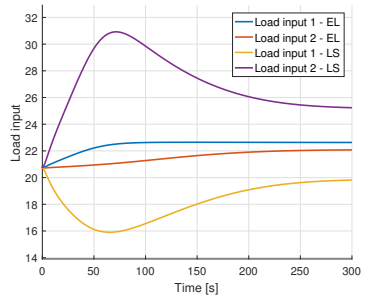

(a) Torque

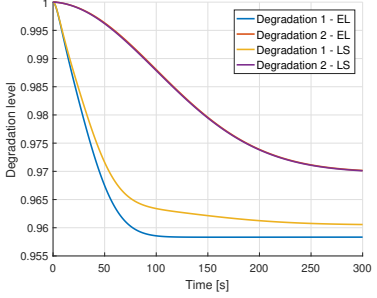

(b) Degradation
Fig. 11: Applied torque and degradation if both compressors degrade

the degradation of the faster degrading compressor (left plot), but, at the same time, it increases the load-induced degradation of the second one.

\section{CONCLUSIONS}

The algorithm proposed in this work is able to mitigate the degradation by reducing the torque applied to the compressor. The results suggest that the best outcome for degradation mitigation is obtained when the degradation rate of one compressor is much slower than the other one. In case if the degradation levels are similar, the ratio is close to unity and the algorithm becomes closer to equal load. It does not require a reconfiguration of lower level controllers, however, the influence of disturbances on the algorithm are to be considered in the future.

Further work includes also a generalization for multiple compressors and the analysis of the degradation indicators. Current degradation model requires knowledge about degradation history to find the values of the parameters. Nonetheless, the parameters might also reflect the operators' knowledge about the process and the influence of the applied control effort on health, i.e. larger $d_{\tau, i}$ means faster overall degradation and will result in lower load on the machine. As both the control requirements and the degradation models are application-specific, the design of a general degradation mitigating control remains an open question.

\section{REFERENCES}

[1] A. Ray, W. M. K., M. Carpino, and C. F. Lorenzo, "Damage-mitigating control of mechanical systems: Part I - conceptual development and 
model formulation," Journal of Dynamic Systems Measurement and Control-transactions of the ASME, vol. 116, no. 3, pp. 437-447, 1994.

[2] BSI, "Bs en iso 20815:2010. petroleum, petrochemical and natural gas industries. production assurance and reliability management," BSI, Tech. Rep., 2010.

[3] O. Härkegård and S. T. Glad, "Resolving actuator redundancy optimal control vs. control allocation," Automatica, vol. 41, no. 1 , pp. 137-144, 2005.

[4] B. G. Liptak, Instrument Engineers' Handbook, Volume Two: Process Control and Optimization. CRC Press, 2005, vol. 2.

[5] S. Staroselsky, B. W. Batson, S. Mirsky, and V. Shapiro, "Method and apparatus for load balancing among multiple compressors," Patent US 5743715 A, 1998.

[6] A. J. Fisher, T. S. Smoot, and D. E. Kincer, "Method and apparatus for controlling a system of compressors to achieve load sharing," Patent US 5343384 A, 1994.

[7] T. Itou and K. Takeda, "Control method of plural compressors and compressor system," Patent US 6773224 B2, 2004.

[8] J. Dirks and P. Jansen, "Method and system for controlling a turbocompressor group," Patent US 20100178154 A1, 2010.

[9] ABB, "SmartSharing ${ }^{\mathrm{TM}}$. Adaptive Load Sharing Controller," 2013, http://www.abb.com/industries/seitp406/ fd28e0438c4dcf2185257c120069ded1.aspx.

[10] SIEMENS, "Siemens load-sharing control," 2011, https: //www.energy.siemens.com/nl/pool/hq/compression/downloads/ SIEMENS_SCOUT_Load_Sharing.pdf.

[11] A. Cortinovis, M. Mercangöz, M. Zovadelli, D. Pareschi, A. De Marco, and S. Bittanti, "Online performance tracking and load sharing optimization for parallel operation of gas compressors," Computers \& Chemical Engineering, vol. 88, pp. 145-156, 52016.

[12] S. Kumar and A. Cortinovis, "Load sharing optimization for parallel and serial compressor stations," in 2017 IEEE Conference on Control Technology and Applications (CCTA). IEEE, 2017, pp. 499-504.

[13] D. P. Xenos, M. Cicciotti, G. M. Kopanos, A. E. F. Bouaswaig, O. Kahrs, R. Martinez-Botas, and N. F. Thornhill, "Optimization of a network of compressors in parallel: Real time optimization (RTO) of compressors in chemical plants - an industrial case study," Applied Energy, vol. 144, pp. 51-63, Apr. 2015.

[14] J. T. Gravdahl, "Modeling and control of surge and rotating stall in compressors," Ph.D. dissertation, 1998, Norwegian University of Science and Technology.

[15] M. P. Boyce, "Principles of operation and performance estimation of centrifugal compressors," in Proceedings of the 22nd Turbomachinery Symposium, 1993, pp. 161-178.

[16] D. Fouflias, A. Gannan, K. Ramsden, P. Pilidis, D. Mba, J. Teixeira, U. Igie, and P. Lambart, "Experimental investigation of the influence of fouling on compressor cascade characteristics and implications for gas turbine engine performance," Proceedings of the Institution of Mechanical Engineers, Part A: Journal of Power and Energy, vol. 224, no. 7, pp. 1007-1018, 2010.

[17] M. Cicciotti, "Adaptive monitoring of health-state and performance of industrial centrifugal compressors," Ph.D. dissertation, 2015, Imperial College London.

[18] A. Tarabrin, V. Schurovsky, A. Bodrov, and J.-P. Stalder, "An analysis of axial compressors fouling and a cleaning method of their blading," in ASME 1996 International Gas Turbine and Aeroengine Congress and Exhibition. American Society of Mechanical Engineers, 1996.

[19] R. Kurz and K. Brun, "Degradation in gas turbine systems," Journal of Engineering for Gas Turbines and Power - Transactions of the ASME, vol. 123, no. 1, pp. 70-77, Jan. 2001.

[20] A. Verheyleweghen and J. Jäschke, "Health-aware operation of a subsea gas compression station under uncertainty," in Foundations of Computer Aided Process Operations /Chemical Process Control, 2017, paper ID F26.

[21] D. Lewandowski, P. Lipnicki, M. Kaczmarek, D. Pareshi, and A. Cortinovis, "Real time model predictive control for centrifugal compressors," in IX International Conference on Computational Heat and Mass Transfer, 2016.

[22] A. Cortinovis, H. J. Ferreau, D. Lewandowski, and M. Mercangöz, "Experimental evaluation of MPC-based anti-surge and process control for electric driven centrifugal gas compressors," Journal of Process Control, vol. 34, pp. 13-25, 2015.

[23] A. Cortinovis, H. J. Ferreau, D. Lewandowski, and M. Mercangoez, "Safe and efficient operation of centrifugal compressors using linearized MPC," in 2014 IEEE 53rd Annual Conference on Decision and Control, 2014, pp. 3982-3987.

[24] O. Egeland and J. T. Gravdahl, Modeling and Simulation for Automatic Control, 1st ed., M. Cybernetics, Ed., 2002. 